\title{
Unstable homotopy invariance for finite fields
}

\author{
by
}

\author{
Kevin P. Knudson (Mississippi State, MS)
}

\begin{abstract}
We prove that if $k$ is a finite field with $p^{d}$ elements, then the natural map $H_{i}\left(\mathrm{GL}_{n}(k), \mathbb{Z}\right) \rightarrow H_{i}\left(\mathrm{GL}_{n}(k[t]), \mathbb{Z}\right)$ is an isomorphism for $0 \leq i<d(p-1)$ and for all $n$.
\end{abstract}

Introduction. In [2] we proved that if $k$ is an infinite field, then for all $n$ the natural map

$$
j_{*}: H_{\bullet}\left(\mathrm{SL}_{n}(k), \mathbb{Z}\right) \rightarrow H_{\bullet}\left(\mathrm{SL}_{n}(k[t]), \mathbb{Z}\right)
$$

induced by the inclusion is an isomorphism. The same result is then true for $\mathrm{GL}_{n}$. The method of proof was to examine the action of $\mathrm{SL}_{n}(k[t])$ on a suitable building $\mathcal{X}$ and then use the resulting spectral sequence. The hypothesis that $k$ be infinite was crucial for the computation of the homology of the various simplex stabilizers.

If $k$ is finite, then one may use the same approach, but the calculation of the homology of the stabilizers is more difficult. In this note, we carry this out and prove the following result.

Theorem 3.1. Let $k$ be a finite field with $p^{d}$ elements. Then for $0 \leq$ $i<d(p-1)$ and for all $n$, the restriction map

$$
j^{*}: H^{i}\left(\mathrm{GL}_{n}(k[t]), \mathbb{Z}\right) \rightarrow H^{i}\left(\mathrm{GL}_{n}(k), \mathbb{Z}\right)
$$

is an isomorphism.

The same result is therefore true for homology. The bound on $i$ is optimal; see Remark 2.2 below.

Note that this result is in keeping with one's intuition that if the field $k$ is large, then the map $j^{*}$ should be an isomorphism "most of the time". Also, it is independent of $n$ and therefore complements what van der Kallen's stability theorem [1] gives:

$$
j^{*}: H^{i}\left(\mathrm{GL}_{n}(k[t]), \mathbb{Z}\right) \rightarrow H^{i}\left(\mathrm{GL}_{n}(k), \mathbb{Z}\right)
$$

2000 Mathematics Subject Classification: Primary 20G10.

Partially supported by NSF grant no. DMS-0070119. 
is an isomorphism for $i \geq 2 n+1$. One may be able to say more about the map $j^{*}$ in specific cases, but Theorem 3.1 seems to be the best possible general result.

The calculation of the cohomology of the stabilizers is similar to Quillen's computation of the mod $p$ cohomology of $\mathrm{GL}_{n}(k)$. Indeed, one need only compute the invariants of the action of the diagonal subgroup $T \subset \mathrm{GL}_{n}(k)$ on the cohomology of various Sylow $p$-subgroups. It was Quillen's paper [3] that motivated the proof of Theorem 3.1.

This paper is organized as follows. In Section 1 we describe the building $\mathcal{X}$ and the stabilizers of the $\mathrm{GL}_{n}(k[t])$ action on it. In Section 2 we calculate the cohomology of the stabilizers. Finally in Section 3 we prove Theorem 3.1.

1. The spectral sequence. Denote by $\mathcal{X}$ the Bruhat-Tits building associated to the vector space $k(t)^{n}$. Recall that the vertices of $\mathcal{X}$ are equivalence classes of $\mathcal{O}$-lattices in $k(t)^{n}$ (here, $\mathcal{O}$ consists of the set of $a / b$ with $\operatorname{deg} b \geq \operatorname{deg} a$ ), where two lattices $L$ and $L^{\prime}$ are equivalent if there is an $x \in k^{\times}$with $L^{\prime}=x L$. A collection of vertices $\Lambda_{0}, \Lambda_{1}, \ldots, \Lambda_{m}$ forms an $m$-simplex if there are representatives $L_{i}$ of the $\Lambda_{i}$ with

$$
t^{-1} L_{0} \subset L_{m} \subset L_{m-1} \subset \ldots \subset L_{0} .
$$

It is possible to put a metric on $\mathcal{X}$ so that each edge in $\mathcal{X}$ has length one. When we speak of the distance between vertices we implicitly use this metric. For a more complete description of $\mathcal{X}$, see, for example, [2].

The group $\mathrm{GL}_{n}(k[t])$ acts on $\mathcal{X}$ with fundamental domain an infinite contractible wedge $\mathcal{T}$, which is the subcomplex of $\mathcal{X}$ spanned by the vertices

$$
\left[e_{1} t^{r_{1}}, \ldots, e_{n-1} t^{r_{n-1}}, e_{n}\right], \quad r_{1} \geq \ldots \geq r_{n-1} \geq 0,
$$

where $e_{1}, \ldots, e_{n}$ denotes the standard basis of $k(t)^{n}$ (see [4]).

Denote by $v_{0}$ the vertex $\left[e_{1}, \ldots, e_{n}\right]$ and by $v_{i}$ the vertex

$$
\left[e_{1} t, \ldots, e_{i} t, e_{i+1}, \ldots, e_{n}\right], \quad i=1, \ldots, n-1 .
$$

For a $q$-element subset $I=\left\{i_{1}, \ldots, i_{q}\right\}$ of $\{1, \ldots, n-1\}$, define $E_{I}^{(q)}$ to be the subcomplex of $\mathcal{T}$ which is the union of all rays with origin $v_{0}$ passing through the $(q-1)$-simplex $\left\langle v_{i_{1}}, \ldots, v_{i_{q}}\right\rangle$. There are $\left(\begin{array}{c}n-1 \\ q\end{array}\right)$ such $E_{I}^{(q)}$. Observe that if $I=\{1, \ldots, n-1\}$, then $E_{I}^{(n-1)}=\mathcal{T}$. When we write $E_{J}^{(l)}$, the superscript $l$ denotes the cardinality of the set $J$.

The structure of the various simplex stabilizers was described in [2]. If $\left(x_{i j}(t)\right) \in \mathrm{GL}_{n}(k[t])$ stabilizes the vertex $\left[e_{1} t^{r_{1}}, \ldots, e_{n-1} t^{r_{n-1}}, e_{n}\right]$, then we have

$$
\operatorname{deg} x_{i j}(t) \leq r_{i}-r_{j}
$$

(set $\left.r_{n}=0\right)$. Note that since $r_{1} \geq \ldots \geq r_{n-1}$, some of the $x_{i j}(t)$ with $i>j$ may be 0 . Denote the stabilizer of $\sigma$ by $\Gamma_{\sigma}$. The group $\Gamma_{\sigma}$ is the intersection 
of the stabilizers $\Gamma_{v}$ where $v$ ranges over the vertices of $\sigma$. In this case $\operatorname{deg} x_{i j}(t) \leq \min _{v \in \sigma}\left\{r_{i}^{(v)}-r_{j}^{(v)}\right\}$. Observe that in any case, the group $\Gamma_{\sigma}$ has a block form where the blocks along the diagonal are matrices with entries in $k$, blocks below are zero, and blocks above contain polynomials of bounded degree. In the case $n=3$, we have the following block forms:

$$
\begin{aligned}
v_{0}: & \Gamma_{v_{0}}=\mathrm{GL}_{3}(k) \\
\sigma \in E_{\{1\}}^{(1)}: & \Gamma_{\sigma}=\left(\begin{array}{c|cc}
* & * & * \\
\hline 0 & * & * \\
0 & * & *
\end{array}\right), \\
\sigma \in E_{\{2\}}^{(1)}: & \Gamma_{\sigma}=\left(\begin{array}{cc|c}
* & * & * \\
& * & * \\
\hline 0 & 0 & *
\end{array}\right), \\
\sigma \in \mathcal{T}-\left(E_{\{1\}}^{(1)} \cup E_{\{2\}}^{(1)}\right): & \Gamma_{\sigma}=\left(\begin{array}{ccc}
* & * & * \\
0 & * & * \\
0 & 0 & *
\end{array}\right) .
\end{aligned}
$$

We have a short exact sequence

$$
1 \rightarrow C_{\sigma} \rightarrow \Gamma_{\sigma} \stackrel{t=0}{\longrightarrow} P_{\sigma} \rightarrow 1
$$

where $P_{\sigma}$ is a parabolic subgroup of $\mathrm{GL}_{n}(k)$. From the above description of $\Gamma_{\sigma}$, we see that the group $C_{\sigma}$ has a block form where blocks along the diagonal are identity matrices, blocks below are zero, and blocks above contain polynomials of bounded degree which are divisible by $t$.

Filter the complex $\mathcal{T}$ by setting $W^{(0)}=v_{0}$ and

$$
W^{(l)}=\bigcup_{|I|=l} E_{I}^{(l)}, \quad 1 \leq l \leq n-1 .
$$

Observe that if $\sigma$ and $\tau$ are simplices in the same component of $W^{(i)}-$ $W^{(i-1)}$, then $P_{\sigma}=P_{\tau}$ since on such a component the relationships among the $r_{i}$ defining the vertices do not vary from vertex to vertex (i.e., if $r_{i}>r_{i+1}$ for one vertex in the component, then the same holds for every vertex in the component; since these relations determine which entries below the diagonal are zero, we see that the stabilizers of these vertices all have the same block form and hence so does the stabilizer of any simplex in the component).

The action of $\mathrm{GL}_{n}(k[t])$ on $\mathcal{X}$ gives rise to a spectral sequence converging to $H^{\bullet}\left(\mathrm{GL}_{n}(k[t]), \mathbb{Z}\right)$ with $E_{1}$-term

$$
E_{1}^{p, q}=\prod_{\operatorname{dim} \sigma=p} H^{q}\left(\Gamma_{\sigma}, \mathbb{Z}\right)
$$


where $\Gamma_{\sigma}$ denotes the stabilizer of the simplex $\sigma \in \mathcal{T}$. Now we shall compute the terms $E_{2}^{p, 0}$.

Proposition 1.1. The bottom row of the spectral sequence (1) satisfies

$$
E_{2}^{p, 0}=0, \quad p>0 .
$$

Proof. The bottom row of the spectral sequence consists of the groups

$$
E_{1}^{p, q}=\prod_{\operatorname{dim} \sigma=p} H^{0}\left(\Gamma_{\sigma}, \mathbb{Z}\right)
$$

This is simply the cochain complex $C^{\bullet}(\mathcal{T}, \mathbb{Z})$. Since $\mathcal{T}$ is contractible, we see that $E_{2}^{p, 0}=0$ for $p>0$.

We now consider cohomology with $\mathbb{F}_{l}$-coefficients, $l \neq p$. In this case we see that since in the extensions

$$
1 \rightarrow C_{\sigma} \rightarrow \Gamma_{\sigma} \rightarrow P_{\sigma} \rightarrow 1
$$

the subgroups $C_{\sigma}$ are $p$-groups, the map $\Gamma_{\sigma} \rightarrow P_{\sigma}$ induces an isomorphism

$$
H^{\bullet}\left(P_{\sigma}, \mathbb{F}_{l}\right) \rightarrow H^{\bullet}\left(\Gamma_{\sigma}, \mathbb{F}_{l}\right) .
$$

Now, for each $q$, we have a coefficient system $\mathcal{H}^{q}$ on $\mathcal{T}$ defined by

$$
\mathcal{H}^{q}(\sigma)=H^{q}\left(\Gamma_{\sigma}, \mathbb{F}_{l}\right) .
$$

In this notation, then, the $q$ th row of the spectral sequence (1) is the cochain complex $C^{\bullet}\left(\mathcal{T}, \mathcal{H}^{q}\right)$. Using the isomorphism (2), we see that on each component of $W^{(i)}-W^{(i-1)}$ the system $\mathcal{H}^{q}$ is constant for each $q$. By Lemma 5 of [4] the inclusion $v_{0} \rightarrow \mathcal{T}$ induces an isomorphism

$$
H^{\bullet}\left(\mathcal{T}, \mathcal{H}^{q}\right) \stackrel{\cong}{\longrightarrow} H^{\bullet}\left(v_{0}, \mathcal{H}^{q}\right)
$$

for all $q$. It follows that

$$
E_{2}^{p q}= \begin{cases}H^{q}\left(\mathrm{GL}_{n}(k), \mathbb{F}_{l}\right), & p=0 \\ 0, & p>0\end{cases}
$$

Hence we have the following result, due to Soulé [4].

Proposition 1.2. If $l \neq p$, then the natural map $\mathrm{GL}_{n}(k) \rightarrow \mathrm{GL}_{n}(k[t])$ induces an isomorphism

$$
H^{\bullet}\left(\mathrm{GL}_{n}(k[t]), \mathbb{F}_{l}\right) \rightarrow H^{\bullet}\left(\mathrm{GL}_{n}(k), \mathbb{F}_{l}\right)
$$

for all $n$.

It remains to calculate the $\mathbb{F}_{p}$-cohomology of the various $\Gamma_{\sigma}$; we do this in the next section. 
2. Mod $p$ cohomology. In this section cohomology is with $\mathbb{F}_{p}$ coefficients. We shall write $H^{\bullet}(G)$ for $H^{\bullet}\left(G, \mathbb{F}_{p}\right)$.

Let $\Gamma_{\sigma}$ be the stabilizer of the simplex $\sigma$ and consider the extension

$$
1 \rightarrow C_{\sigma} \rightarrow \Gamma_{\sigma} \rightarrow P_{\sigma} \rightarrow 1,
$$

where $P_{\sigma}$ is a parabolic subgroup of $\mathrm{GL}_{n}(k)$. Observe that the Sylow $p$ subgroup of $P_{\sigma}$ is the group $U_{n}(k)$ consisting of upper triangular unipotent matrices over $k$. Indeed, if $Q$ is a Sylow $p$-subgroup of $P_{\sigma}$, then $Q \supseteq U_{n}(k)$ since $U_{n}(k)$ is a $p$-subgroup of $P_{\sigma}$. But since $Q$ is a $p$-subgroup of $\mathrm{GL}_{n}(k)$, we must have $Q \subseteq U_{n}(k)$. Thus, denoting by $U_{\sigma}$ a Sylow $p$-subgroup of $\Gamma_{\sigma}$, we have an extension

$$
1 \rightarrow C_{\sigma} \rightarrow U_{\sigma} \rightarrow U_{n}(k) \rightarrow 1 .
$$

The group $U_{\sigma}$ consists of matrices whose $i, j$ entry has degree less than $l_{i j}$, where

$$
l_{i j}=\min _{v \in \sigma}\left\{r_{i}^{(v)}-r_{j}^{(v)}\right\}
$$

(see Section 1).

Now, the restriction map

$$
j^{*}: H^{\bullet}\left(\Gamma_{\sigma}\right) \rightarrow H^{\bullet}\left(U_{\sigma}\right)
$$

is injective. If $T$ denotes the diagonal subgroup of $\mathrm{GL}_{n}(k)$, then since $T$ normalizes $U_{\sigma}$, the image of $j^{*}$ lies in the subgroup of $T$-invariants.

Proposition 2.1. $H^{i}\left(U_{\sigma}\right)^{T}=0$ for $0<i<d(p-1)$.

Proof. Let $\Delta^{+}$be the set of positive roots $\left(t \mapsto t_{i} / t_{j}, 1 \leq j<i \leq n\right.$, where $t_{i}$ is the $i$ th entry of the diagonal matrix $t$ ). Order $\Delta^{+}$by setting $\left(i^{\prime}, j^{\prime}\right) \leq(i, j)$ if either $i^{\prime}<i$, or $i^{\prime}=i$ and $j^{\prime} \leq j$. If $a \in \Delta^{+}$, let $U_{a}$ be the subgroup of $U_{\sigma}$ generated by the one-parameter subgroups corresponding to roots $>a$. For each $a \in \Delta^{+}$, we have an extension

$$
1 \rightarrow\left(k_{a}\right)^{l_{a}} \rightarrow U_{\sigma} / U_{a} \rightarrow U_{\sigma} / U_{a^{\prime}} \rightarrow 1,
$$

where $k_{a}$ is the $T$-module $k$ with $T$ acting via the root $a, l_{a}=l_{i j}$ for $a=(i, j)$, and $a^{\prime}$ is the element of $\Delta^{+}$immediately preceding $a$ (if $a=(1,2)$, then $\left.U_{a^{\prime}}=U_{\sigma}\right)$. The group $T$ acts on this extension. Since the extension is central, the associated Hochschild-Serre spectral sequence has the form

$$
E_{2}=H^{\bullet}\left(U_{\sigma} / U_{a^{\prime}}\right) \otimes H^{\bullet}\left(k_{a}\right)^{\otimes l_{a}} \Rightarrow H^{\bullet}\left(U_{\sigma} / U_{a}\right) .
$$

Denote by $\operatorname{PS}(M)$ the Poincaré series of the $T$-module $M$. By Lemma 15 of [3], we have

$$
\operatorname{PS}\left(H^{\bullet}\left(k_{a}\right)\right)=\prod_{b=0}^{d-1}\left(1+a^{-p^{b}} z\right) /\left(1-a^{-p^{b}} z^{2}\right) .
$$


The spectral sequence (3) shows that

$$
\operatorname{PS}\left(H^{\bullet}\left(U_{\sigma} / U_{a}\right)\right) \ll \operatorname{PS}\left(H^{\bullet}\left(U_{\sigma} / U_{a^{\prime}}\right)\right) \operatorname{PS}\left(H^{\bullet}\left(k_{a}\right)\right)^{l_{a}},
$$

where $\ll$ means that each term in the series on the left is less than or equal to the corresponding term on the right. Combining these for all $a \in \Delta^{+}$, we have, letting $M=\max _{a \in \Delta+}\left\{l_{a}\right\}$,

$$
\begin{aligned}
\operatorname{PS}\left(H^{\bullet}\left(U_{\sigma}\right)\right) & \ll \prod_{a \in \Delta^{+}} \operatorname{PS}\left(H^{\bullet}\left(k_{a}\right)\right)^{l_{a}} \ll \prod_{a \in \Delta^{+}} \operatorname{PS}\left(H^{\bullet}\left(k_{a}\right)\right)^{M} \\
& =\left[\prod_{a \in \Delta^{+}} \prod_{b=0}^{d-1}\left(1+a^{-p^{b}} z\right) \sum_{j \geq 0} a^{-j p^{b}} z^{2 j}\right]^{M} \\
& =\left[\sum_{I}\left(\prod_{a \in \Delta^{+}} a^{-M_{a}(I)}\right) z^{D(I)}\right]^{M}
\end{aligned}
$$

where $I$ runs over families $\left(m_{a b}, n_{a b}\right)$ with $0 \leq m_{a b} \leq 1$ and $n_{a b} \geq 0$, and

$$
M_{a}(I)=\sum_{b=0}^{d-1}\left(m_{a b}+n_{a b}\right) p^{b}, \quad D(I)=\sum_{a \in \Delta^{+}} \sum_{b=0}^{d-1}\left(m_{a b}+2 n_{a b}\right) .
$$

It follows that

$$
\operatorname{PS}\left(H^{\bullet}\left(U_{\sigma}\right)\right) \ll \sum_{r \geq 0}\left(\sum_{I_{1}, \ldots, I_{M}: \sum D\left(I_{j}\right)=r}\left(\prod_{a \in \Delta^{+}} a^{-\sum_{j=1}^{M} M_{a}\left(I_{j}\right)}\right)\right) z^{r} .
$$

Now, it suffices to show that if $I_{1}, \ldots, I_{M}$ satisfy

$$
\prod_{a \in \Delta^{+}} a^{-\sum_{j=1}^{M} M_{a}\left(I_{j}\right)}=\varepsilon
$$

where $\varepsilon$ is the trivial character of $T$, then

$$
\sum_{j=1}^{M} D\left(I_{j}\right) \geq d(p-1)
$$

Let $a_{i}(t)=t_{i+1} / t_{i}, 1 \leq i \leq n-1$, be the simple roots. Then if $a=$ $t_{j} / t_{h} \in \Delta^{+}$, we may write

$$
a=\prod_{i=1}^{n-1}\left(a_{i}\right)^{c_{a i}}
$$

where $c_{a i}=1$ for $h \leq i<j$ and 0 otherwise. Then our equation becomes

$$
\prod_{a} a^{-\sum_{j=1}^{M} M_{a}\left(I_{j}\right)}=\prod_{i=1}^{n-1} a_{i}^{-c_{a i} \sum_{j=1}^{M} M_{a}\left(I_{j}\right)}=\prod_{i=1}^{n-1} a_{i}^{-e_{i}}
$$


where

$$
e_{i}=c_{a i} \sum_{j=1}^{M} \sum_{b=0}^{d-1}\left(m_{a b}^{(j)}+n_{a b}^{(j)}\right) p^{b}
$$

and $I_{j}=\left\{\left(m_{a b}^{(j)}, n_{a b}^{(j)}\right)\right\}$.

Since $k^{\times}$is cyclic of order $p^{d}-1$, for each $1 \leq i<n$ we have

$$
\sum_{j=1}^{M} \sum_{b=0}^{d-1} c_{a i}\left(m_{a b}^{(j)}+n_{a b}^{(j)}\right) p^{b} \equiv 0 \bmod \left(p^{d}-1\right)
$$

and hence

$$
\sum_{b=0}^{d-1} \sum_{j=1}^{M} c_{a i}\left(m_{a b}^{(j)}+n_{a b}^{(j)}\right) p^{b} \equiv 0 \bmod \left(p^{d}-1\right) .
$$

By Lemma 16 of [3], we have

$$
\sum_{b=0}^{d-1} \sum_{j=1}^{M} c_{a i}\left(m_{a b}^{(j)}+n_{a b}^{(j)}\right) \geq d(p-1)
$$

provided at least one of the $\sum_{j} c_{a i}\left(m_{a b}^{(j)}+n_{a b}^{(j)}\right)$ is nonzero.

Now, suppose that for some $j, D\left(I_{j}\right)>0$. Then for some $a, b$, we have $m_{a b}+n_{a b}>0$ and hence $\sum_{j} c_{a i}\left(m_{a b}^{(j)}+n_{a b}^{(j)}\right)>0$ for some $b$ and $i$. Thus,

$$
\begin{aligned}
\sum_{j=1}^{M} D\left(I_{j}\right) & =\sum_{j=1}^{M}\left(\sum_{a, b}\left(m_{a b}^{(j)}+2 n_{a b}^{(j)}\right)\right) \geq \sum_{j=1}^{M}\left(\sum_{a, b}\left(m_{a b}^{(j)}+n_{a b}^{(j)}\right)\right) \\
& \geq \sum_{j=1}^{M}\left(\sum_{a, b} c_{a i}\left(m_{a b}^{(j)}+n_{a b}^{(j)}\right)\right) \geq d(p-1)
\end{aligned}
$$

as required.

REMARK 2.2. Note that this bound is optimal. Indeed, if $k=\mathbb{F}_{p}, \sigma=v_{0}$, then $\Gamma_{\sigma}=\mathrm{GL}_{n}(k), U_{\sigma}=U_{n}(k)$, and $H^{p-1}\left(U_{n}(k)\right)$ contains $H^{1}\left(k_{a}\right)^{\otimes(p-1)}$, which is a trivial $T$-module by Fermat's Little Theorem. Another example is provided by the group $\mathrm{GL}_{2}\left(\mathbb{F}_{2}[t]\right)$. Using the amalgamated free product decomposition of this group, one can show that $H_{1}\left(\mathrm{GL}_{2}\left(\mathbb{F}_{2}[t]\right), \mathbb{Z}\right)$ contains an infinite-dimensional $\mathbb{F}_{2}$-vector space, while $H_{1}\left(\mathrm{GL}_{2}\left(\mathbb{F}_{2}\right), \mathbb{Z}\right)$ is finite.

\section{The main theorem}

TheOREm 3.1. If $0 \leq i<d(p-1)$, then the restriction map

$$
j^{*}: H^{i}\left(\mathrm{GL}_{n}(k[t]), \mathbb{Z}\right) \rightarrow H^{i}\left(\mathrm{GL}_{n}(k), \mathbb{Z}\right)
$$

is an isomorphism. 
Proof. We know that $j^{*}$ is an isomorphism for all $i$ with $\mathbb{F}_{l^{-}}$coefficients, and for $i=0$ with $\mathbb{Z}$-coefficients. Let us consider $\mathbb{F}_{p}$-cohomology. By Proposition 2.1, the $E_{1}$-term of the spectral sequence (1) satisfies

$$
E_{1}^{p, q}=0, \quad 0<q<d(p-1) .
$$

It follows then that $E_{\infty}^{p, q}=0$ for $0<q<d(p-1)$. Since $E_{\infty}^{p, 0}=0$ for $p>0$, we see that

$$
H^{i}\left(\mathrm{GL}_{n}(k[t]), \mathbb{F}_{p}\right)=0, \quad 0<i<d(p-1) .
$$

Since the same is true for $H^{i}\left(\mathrm{GL}_{n}(k), \mathbb{F}_{p}\right)$ (see [3]), we conclude that $j^{*}$ is an isomorphism for $0 \leq i<d(p-1)$.

\section{References}

[1] W. van der Kallen, Homology stability for linear groups, Invent. Math. 60 (1980), 269-295.

[2] K. Knudson, The homology of special linear groups over polynomial rings, Ann. Sci. École Norm. Sup. (4) 30 (1997), 385-415.

[3] D. Quillen, On the cohomology and $K$-theory of the general linear groups over a finite field, Ann. of Math. (2) 96 (1972), 552-586.

[4] C. Soulé, Chevalley groups over polynomial rings, in: Homological Group Theory, C. T. C. Wall (ed.), London Math. Soc. Lecture Note Ser. 36, Cambridge Univ. Press, Cambridge, 1979, 359-367.

Department of Mathematics and Statistics

Mississippi State University

P.O. Drawer MA

Mississippi State, MS 39762, U.S.A.

E-mail: knudson@math.msstate.edu 